\title{
PION-NUCLEON SCATTERING LENGTHS IN THE CLOUDY BAG MODEL
}

\author{
B.K. JENNINGS, E.A. VEIT ${ }^{1}$ \\ TRIUMF, 4004 Wesbrook Mall, Vancouver, B.C., Canada V6T $2 A 3$
}

and

\author{
A.W. THOMAS \\ Department of Physics, University of Adelaide, South Australia 5001, Australia
}

Received 20 June 1984

\begin{abstract}
We re-examine pion-nucleon s-wave scattering in the volume coupling version of the CBM. If the effects of multiple scattering are taken into account, one needs some phenomenological repulsive interaction in order to reproduce the experimental data. We parametrize this repulsion as a twenty percent increase in the pion mass inside the bag. It is argued that this relatively small change in mass provides strong, a posteriori support for the original assumptions of the CBM.
\end{abstract}

While the MIT bag model [1-3] combines the key features expected in QCD, namely confinement and asymptotic freedom, in a successful, phenomenological package, it also breaks chiral symmetry [3-5]. A number of phenomenological extensions have been developed recently, which restore chiral symmetry by explicitly introducing the pion as a Goldstone boson [3, 6-8]. At the present time there is little agreement over which of these diverse hybrid models (if any, see refs. [9-11]) is closer to the truth, and on that point we do not intend to take a stand here.

We begin with the observation that one of the major successes of current algebra in the late 60's was to give a fairly deep understanding of low energy, s-wave $\pi \mathrm{N}$ scattering through the Weinberg-Tomozawa relationship [12]. It is therefore natural to ask how this relationship is realized in any model which claims to respect PCAC. One of the frustrations in the early development of the cloudy bag model (CBM) was that it did not provide a transparent explanation of the $\pi \mathrm{N}$ scattering lengths. However, in 1981 several groups $[13,14]$ independently showed that a new version of

1 Permanent address (after September 1, 1984): Departamento de Fisica, U.F.R.G.S., Rua Luiz Englert, S/N, 90000, Porto Alegre, Brazil. the CBM (which could be obtained by a unitary transformation of the quark fields), in which the pions coupled to the quarks through derivative coupling over the whole bag volume, did give the WeinbergTomozawa result. The transformed lagrangian density has the form

$$
\begin{aligned}
& \mathcal{L}^{\prime}(x)=(\mathrm{i} \overline{\mathrm{q}} \partial \mathrm{q}-B) \theta_{\mathrm{v}}-\frac{1}{2} \overline{\mathrm{q}} \mathrm{q} \delta_{\mathrm{s}} \\
& \quad-\left(\theta_{\mathrm{v}} / 4 f^{2}\right) \overline{\mathrm{q}} \gamma^{\mu} \lambda \mathrm{q} \cdot\left(\phi \times \partial_{\mu} \phi\right) \\
& \quad+\left(\theta_{\mathrm{v}} / 2 f\right) \overline{\mathrm{q}} \gamma^{\mu} \gamma_{5} \lambda \mathrm{q} \cdot \partial_{\mu} \phi+\frac{1}{2}\left(\partial_{\mu} \phi\right)^{2}-\frac{1}{2} m_{\pi}^{2} \phi^{2},
\end{aligned}
$$

where as usual $q$ and $\phi$ describe quark and pion fields, $\theta_{\mathrm{v}}$ is one inside and zero outside the bag, and $\delta_{\mathrm{s}}$ a surface $\delta$-function.

We shall not dwell on the possible physical interpretations of this new version of the CBM [15]. However, there are three points which should be made explicitly. First, the transformation from surface to volume coupling relied on the CBM hypothesis that the pion looks very much like a free pion inside the bag. Second, the four-point, or contact term in eq. (1) was the piece predominantly responsible for s-wave $\pi \mathrm{N}$ scattering. (In fact it has recently been proven by Jennings and Maxwell [15] that both versions of the CBM yield the same $\pi \mathrm{N}$ scattering amplitude at the tree level. However, the convergence properties of the 
volume coupling version are far superior.) Third, the model only yielded the Weinberg-Tomozawa result in Born approximation.

In the past year or so we have extended the volume coupling version of the $\mathrm{CBM}$ to $\mathrm{SU}(3) \times \mathrm{SU}(3)$, and used it to examine the $\overline{\mathrm{K}} \mathrm{N}-\Sigma \pi$ system near $\overline{\mathrm{K}} \mathrm{N}$ threshold [16]. Our rather surprising result was that the $\Lambda^{\prime}(1405)$ is predominantly a $\bar{K} N$ bound-state generated by the contact term. While this is an attractive result for bag model spectroscopy $[17,18]$, the importance of multiple scattering through the contact term in the $S=-1$ system led us to reexamine s-wave $\pi \mathrm{N}$ scattering. Our results were obtained by solving the relativistic Lippmann-Schwinger equation

$$
\begin{aligned}
& t\left(\boldsymbol{k}, \boldsymbol{k}^{\prime} ; E\right)=v\left(\boldsymbol{k}, \boldsymbol{k}^{\prime}\right) \\
& \quad+\int \mathrm{d} \boldsymbol{k}^{\prime \prime} \frac{v\left(\boldsymbol{k}, \boldsymbol{k}^{\prime \prime}\right) t\left(\boldsymbol{k}^{\prime \prime}, \boldsymbol{k}^{\prime} ; E\right)}{E+\mathrm{i} \epsilon-\omega\left(k^{\prime \prime}\right)-E_{N}\left(k^{\prime \prime}\right)},
\end{aligned}
$$

using the contact interaction

$$
\begin{aligned}
& v^{I}\left(\boldsymbol{k}, \boldsymbol{k}^{\prime}\right)=\frac{\lambda^{I} N_{\mathrm{s}}^{2}\left[\omega(k)+\omega\left(k^{\prime}\right)\right]}{(2 \pi)^{3}\left[2 \omega(k) 2 \omega\left(k^{\prime}\right)\right]^{1 / 2}} \\
& \quad \times \int_{0}^{R} \mathrm{~d} r r^{2}\left[j_{0}^{2}\left(\omega_{\mathrm{s}} r / R\right)+j_{1}^{2}\left(\omega_{\mathrm{s}} r / R\right)\right] j_{0}(k r) j_{0}\left(k^{\prime} r\right),
\end{aligned}
$$

which follows from eq. (1) [16]. (Here $\lambda^{I}=+1 / 2$ for $I=3 / 2$ and -1 for $I=1 / 2, N_{\mathrm{s}}$ is the normalization constant for the $1 \mathrm{~s}_{1 / 2}$ bag wave function of eigenfrequency $\omega=2.04 \ldots$.)

From the results given in the first three lines of table 1 we see that there is a large, attractive, isoscalar scattering length in contradiction with the experimental result [20]. Since the isoscalar scattering length is zero in Born approximation, it is clear that the multiple scattering has generated a large unwanted attraction. As a matter of interest we observe that while these results were obtained from the contact interaction, they are very close, both in isospin structure and the actual numbers, to what one would find from $\rho$ meson exchange in the more conventional meson exchange picture.

Phenomenologically it is apparent that a relatively small repulsive interaction would resolve the problem. For example, it would suffice to exclude the pion from a region of radius $\left(R_{1}\right)$ about $0.1 \mathrm{fm}$ at the bag centre
Table 1

Pion-nucleon scattering lengths obtained with a bag radius of $1 \mathrm{fm}$, for various values of the parameters $f$ and $\delta m_{\pi}$ described in the text.

\begin{tabular}{llll}
\hline \multicolumn{1}{l}{} & $\delta m$ & $a_{1}+2 a_{3}(\mathrm{fm})$ & $a_{1}-a_{2}(\mathrm{fm})$ \\
\hline 93 & - & 0.25 & 0.51 \\
100 & - & 0.17 & 0.41 \\
110 & - & 0.11 & 0.31 \\
93 & 27 & 0.02 & 0.42 \\
94 & 35 & -0.05 & 0.38 \\
97 & 28 & -0.01 & 0.37 \\
& & & \\
Exp. A & & $0.01 \pm 0.01$ & $0.43 \pm 0.01$ \\
Exp. B b) & & -0.05 & 0.38 \\
\hline
\end{tabular}
a) Ref. [19].
b) Ref. [20].

$[21,22]$. Such a model has been applied, for example, to the calculation of the axial form factor of the nucleon, but no significant constraint on $\left(R_{1} / R\right)$ was obtained [23]. Alternatively, following Shuryak [24] one could imagine excluding the pion from a cavity (again about $0.1 \mathrm{fm}$ in radius) surrounding each quark.

The less drastic proposal which we investigate here, which is more in the spirit of the original CBM, is to imagine that instead of being completely free the pion has an effective mass $\left(m_{\pi}+\delta m_{\pi}\right)$ inside the bag. By varying $\delta m_{\pi}$ and $f$ we obtain the results shown in table 1 . Uncertainties in the experimental values preclude a unique determination of $\delta m_{\pi}$ but we see that it is of the order of $30 \mathrm{MeV}$. Although $f$ was allowed to vary freely, the fact that it is so near the theoretically expected value of $96 \mathrm{MeV}$ (calculated as $93 \mathrm{MeV}$ modified by renormalization effects [16]) is a reassuring check on the consistency of the model.

Before commenting on the significance of $\delta m_{\pi}$ we should ask what other contributions to s-wave scattering could arise in this model. Scattering through the observed negative parity baryon resonances is very small $(<0.01 \mathrm{fm})$ because of their large masses. We feel that it would be inconsistent to include the exchange of a fictitious scalar meson [25]. One would certainly find a repulsive contribution from scattering through antibaryon intermediate states [25], which would eliminate most of the need for $\delta m_{\pi}$. However, in a model with composite baryons one might expect such terms to be strongly suppressed [26]. Finally, one might consider scattering via antiparticle states at the quark 
level i.e. $4 q-\bar{q}$ states. Jennings and Maxwell [15] found a contribution of $-0.03 \mathrm{fm}$ in Born approximation (for $a_{1}+2 a_{3}$ ) from these, which would account for about $50 \%$ of $\delta m_{\pi}$. However, as with the particle states in their model, this is probably an overestimate. In any case, the sign of these contributions is such as to strengthen our conclusions concerning $\delta m_{\pi}$.

To summarize, we have shown that the volume coupling version of the CBM can describe low energy $\pi \mathrm{N}$ scattering, provided the pion feels a relatively small repulsive force inside the bag $\left(\delta m_{\pi} \sim 30 \mathrm{MeV}\right)$. What is most surprising about this result is not the fact that such repulsion exists, but that it is so small! If, as proposed by De Tar [8] for example, allowing pions in the bag was a crude representation of the propagation of uncorrelated $\mathrm{q} \bar{q}$ pairs with pion quantum numbers, one would naively expect $\delta m_{\pi}$ to be of order $800 \mathrm{MeV}$ $(\sim 2 \omega / R)$. Our finding that $\delta m_{\pi}$ is instead so near to zero provides strong phenomenological evidence that the correlation between such pairs is indeed strong, and as a first approximation it makes sense to talk instead of a free pion inside the bag.

Our conclusions are based on a model where the radius is sufficiently large that the pions can be treated perturbatively. It would be interesting if proponents of various alternative hybrid bag models could test their models against low energy s-wave $\pi \mathrm{N}$ scattering.

This work has been partially supported by NSERC (Canada), CNPq and FINEP (Brazil).

\section{References}

[1] A. Chodos, R.L. Jaffe, K. Johnson, C.B. Thorn and V.F. Weisskopf, Phys. Rev. D9 (1974) 3471;

A. Chodos, R.L. Jaffe, K. Johnson and C.B. Thorn, Phys. Rev. D10 (1974) 2599.
[2] C.E. De Tar and J.F. Donoghue, Ann. Rev. Nucl. Part. Sci. 33 (1983) 235.

[3] A.W. Thomas, Adv. Nucl. Phys. 13 (1984) 1.

[4] A. Chodos and C.B. Thorn, Phys. Rev. D12 (1975) 2733.

[5] T. Inoue and T. Maskawa, Prog. Theor. Phys. 54 (1975) 1833.

[6] G.E. Brown and M. Rho, Phys. Lett. 82B (1979) 177; V. Vento, M. Rho, E.M. Nyman, J.H. Jun and G.E. Brown, Nucl. Phys. A345 (1980) 413.

[7] S. Théberge, A.W. Thomas and G.A. Miller, Phys. Rev. D22 (1980) 2838; D23 (1981) 2106 (E); Can. J. Phys. $60(1982) 59$.

[8] C. De Tar, Phys. Rev. D24 (1981) 752, 762.

[9] T.H.R. Skyrme, Proc. R. Soc. A260 (1961) 127.

[10] E. Witten, Nucl. Phys. B160 (1979) 57; B223 (1983) $422,433$.

[11] M. Rho, A.S. Goldhaber and G.E. Brown, Phys. Rev. Lett. 51 (1983) 747; J. Goldstone and R.L. Jaffe, Phys. Rev. Lett. 51 (1983) 747.

[12] S. Weinberg, Phys. Rev. Lett. 17 (1966) 616; 18 (1967) 188.

[13] A.W. Thomas, J. Phys. G7 (1981) L283.

[14] A. Szymacha and S. Tatur, Z. Phys. C7 (1981) 311.

[15] B.K. Jennings and O.V. Maxwell, Nucl. Phys,, to be published.

[16] E.A. Veit, B.K. Jennings, R.C. Barrett and A.W. Thomas, Phys. Lett. 137B (1984) 415 ; and TRIUMF preprint TRI-PP-84-40, to be published.

[17] T.A. De Grand, Ann. Phys. 101 (1976) 496.

[18] E.A. Veit, B.K. Jennings, R.C. Barrett and A.W. Thomas, contributed paper 10th Intern. Conf. on Particles and nuclei (Heidelberg, 1984).

[19] V.S. Zidell, R.N. Arndt and L.D. Roper, Phys. Rev. D21 (1980) 1289.

[20] J. Stahov, Karlsruhe preprint TKP82-16.

[21] V. Vento, Phys. Lett. 121B (1983) 370.

[22] S.A. Chin and G.A. Miller, Phys. Lett. 121B (1983) 232.

[23] P.A.M. Guichon, G.A. Miller and A.W. Thomas, Phys. Lett. 124B (1983) 109.

[24] E.V. Shuryak, CERN yellow report 83-01.

[25] F. Hachenberg and H.J. Pirner, Ann. Phys. (NY) 112 (1978) 401.

[26] S.J. Brodsky and J.R. Hiller, Phys. Rev. C28 (1983) 475. 